\title{
EVALUATION THE AFFECTS OF MIMO BASED RAYLEIGH NETWORK CASCADED WITH UNSTABLE DEAD ZONE SATURATION SYSTEM
}

\author{
Nirmalya Chandra ${ }^{1}$, Samiran Maiti ${ }^{2}$, Achintya Das $^{3}$ \\ ${ }^{1}$ Assistant Professor, Birbhum Institute of Engineering and Technology, Suri, Birbhum, West Bengal, Pin 731101 \\ ${ }^{2}$ Assistant Professor, Birbhum Institute of Engineering and Technology, Suri, Birbhum, West Bengal, Pin 731101 \\ ${ }^{3}$ Professor \& Head of Dept. of Electronics and Communication Engineering, Kalyani Govt. Engineering College, \\ P.O: Kalyani; Dist: Nadia, West Bengal, PIN: 741 235; India. ${ }^{3}$
}

\begin{abstract}
Nonlinear Control is the area of control engineering specifically involved with systems that are nonlinear, time-variant, or both. Many well-established analysis and design techniques exist for linear time-invariant (LTI) systems. However, one or both of the controller and the system under control in a general control system may not be an LTI system, and so these methods cannot necessarily be applied directly. Nonlinear control theory studies how to apply existing linear methods to these more general control systems. In this paper we generalized a MIMO based Rayleigh network where by diversity technique we improve the signal to noise ratio and see the effects if its passing through a dead zone saturation system. We also see the effects of Zero Order Hold technique to produce a smooth running system.
\end{abstract}

Keywords: Dead Zone Saturation System, MRC Diversity Technique, ABER, Zero Order Hold Technique ***

\section{INTRODUCTION}

A system always follows some rules. Whenever we try to describe system for the timbering we generally establish a relation between output and input part of the system. For easy way, if, among the twos one of a system must follows linearity and homogeneity then by analysis on processing function of the system it would be try to find out the stability point of system. These happens for only linear system.

But naturally system are non linear in practice where the above stated principle are not applicable. For example, for a sinusoidal input, linear system demands output obviously follows input i.e sinusoidal in nature but in practice, the output signal may content so many harmonics and sub-harmonics of various unexpected amplitudes and phase difference. So this characteristics obviously unbounded and exist the properties of limit cycle. The output state of an unstable non; linear system can go to infinity time.

So, the general model [ 1 ] of non linear system is as follows :

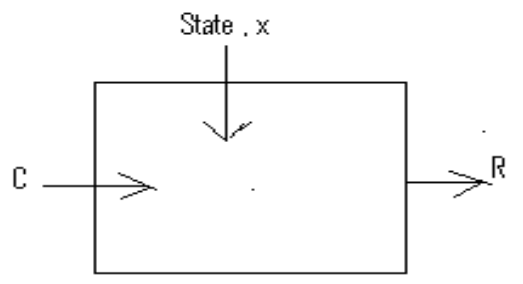

Fig 1: General Model of Non linear system

$\mathrm{x}^{\prime}=f(\tau, \mathrm{x}, \mathrm{C}, \mathrm{W}):$ State Equation

$\mathrm{R}=h(\tau, \mathrm{x}, \mathrm{C}, \mathrm{W}):$ Response Equation

Where, $x \in D^{i} ; C € D^{j} ; W € D^{k}$

Here, ' $\mathrm{x}$ ' is the state of the system and ' $\mathrm{W}$ ' is the Creating Disturbance ; $\mathrm{C}$ is the input and $\mathrm{R}$ is the response ( output ) of the system ; D is the dynamical closed loop system ; $\mathrm{i}, \mathrm{j}, \mathrm{k}$ are the vector notation which trace the system in a well defined form.

In some cases [ 2 ] disturbance factor is not consider i.e $\mathrm{x}^{\prime}=f$ $(\tau, \mathrm{x}, \mathrm{C})$ and as well as even the system does not depend on input $\mathrm{C}$, then its behave as an unforced system where the input not indicates any separate 
Now, for an infinite dimensional system, the system is described with the help of distributed parameters i.e in that case, $f(\tau, \mathrm{x}, \mathrm{C})=0$

Where as a linear system can be modeled as:

$$
R_{L}(\tau)=W_{0 L}(\tau) C_{L}(\tau)+\int_{0}^{\tau} W_{1 L}(\tau, \Delta \tau) C_{L}(\Delta \tau) d \Delta \tau \ldots
$$

Whereas, a Non Linear System can be modeled as :

$$
\begin{aligned}
& R_{N L}(\tau)=W_{0 N L}(\tau) C_{N L}(\tau)+\int_{0}^{\tau} W_{1 N L}(\tau, \Delta \tau) C_{N L}(\Delta \tau) d \Delta \tau \\
& +\int_{0}^{\tau} \int_{0}^{\Delta \tau 1} W_{2 N L}(\tau, \Delta \tau 1, \Delta \tau 2) C_{N L}(\Delta \tau 2) d \Delta \tau 1 d \Delta \tau 2+\ldots
\end{aligned}
$$

characteristics its itself as a function of $\tau\{\mathrm{C}=f(\tau)\}$. But if $\mathrm{x} '=f(\mathrm{x})$ only then it's a time invariantsystem which tends to force time to reach in origin.

\section{CLASSIFICATION}

In Broad sense [ 3 ] non satisfied superposition principle can be explained by two categories.

First one is very close to linear characteristics. Here, the problem of nonlinearity can be smoothly converted into linear problem by feedback linearization method.

On the other hand if reaching of stable point is very difficult, it indicates the another one. Here the nonlinearity problem can be produced in terms of screendipitous, cautions, evolutionary, tactical.

- Screendipitious the problem of constraints violets occasionally but no precautions are taken advance to avoid it.

- Cautions plays the roll in case of low gain controller system in which the objective is to produce closed loop asymptotic stability in bounded system. But at the cost of incurring performance is mitigated.

- Evolutionary is basically used in antiwindup controller. Here the broadly affections of constraints are suppressed, basically used in industry.

- Tactial is basically a model of predictive control where by chemical process the effect of constraints has to be reduced.
Stability basically has been seen [ 4 ] is linear system. For example for a second order system, the damping ratio $(\xi)$ indicated by radiating area shown in fig. 2 below depicts the minimum covering area, $\xi=\cos \theta$ or $\sin \varphi$

The condition for stable system must be satisfied the following

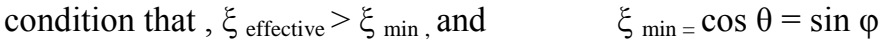
$=\xi$, where $\theta+\varphi=\pi / 2$

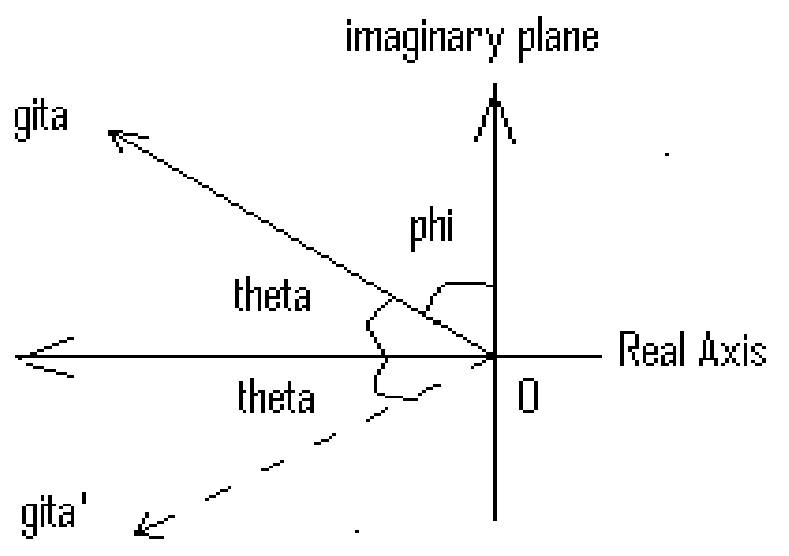

Fig 2 : Plot of radiating area of a Damping Ratio

Now, evaluation of roots for a processing function of a system on either side of numerator or denominator indicates if zeros and poles that plays a vital role in addition case of there.

Study Case 1: $\frac{k}{s(s+a)}=\frac{a d d}{\text { pole }}>\frac{k}{s(s+a)(s+b)}=$

\{Assuming, $|c|>|b|>|a|\} \frac{\text { add }}{\text { pole }}>\frac{1}{s(s+a)(s+b)(s+c)}$

Here the more roots added to increase the no of poles, the root locus goes through unstable position that means go through right handed side of origin shown below:

\section{STABILITY ANALYSIS}


3.1 'Root Locus of G(S) of Single Pole: In the Left Hand Side Hence the System is Stable for all Values of $\mathbf{K}^{\prime}$

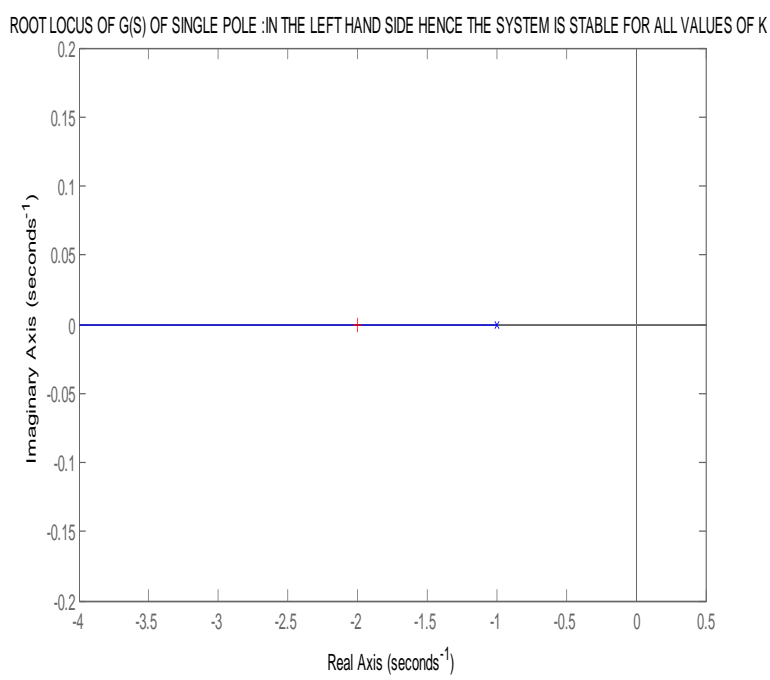

Select a point in the graphics window

selected_point $=$ Empty matrix: 0-by-1 : 1

\section{2 'Root Locus of G(S) with Two Pole on the LHS of}

S Plane: In the Left Hand Side hence the System is

\section{Stable for all Values of $K^{\prime}$}

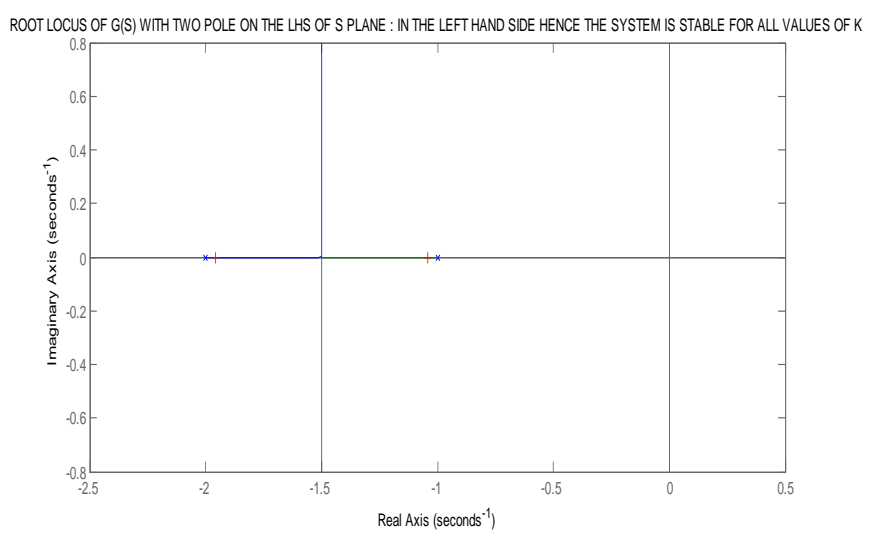

Select a point in the graphics window

selected_point $=-1.0178+0.0373 \mathrm{i}: 0.0406$

\section{3 'Root Locus of $G(S)$ with Increase of No of Poles} Crosses the Imaginary Axix : Stability -> Unstability'

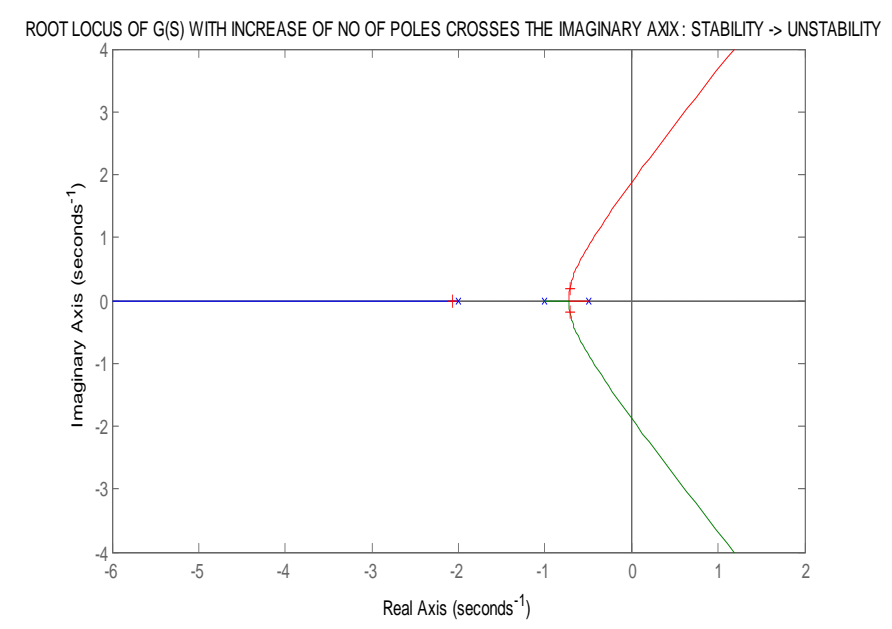

Select a point in the graphics window

selected_point $=-1.2133-0.0373 \mathrm{i}: \quad 0.1218$

\section{4 'Root Locus OG G(S) of Adding Complex Pole: System Tends to Restricted'}

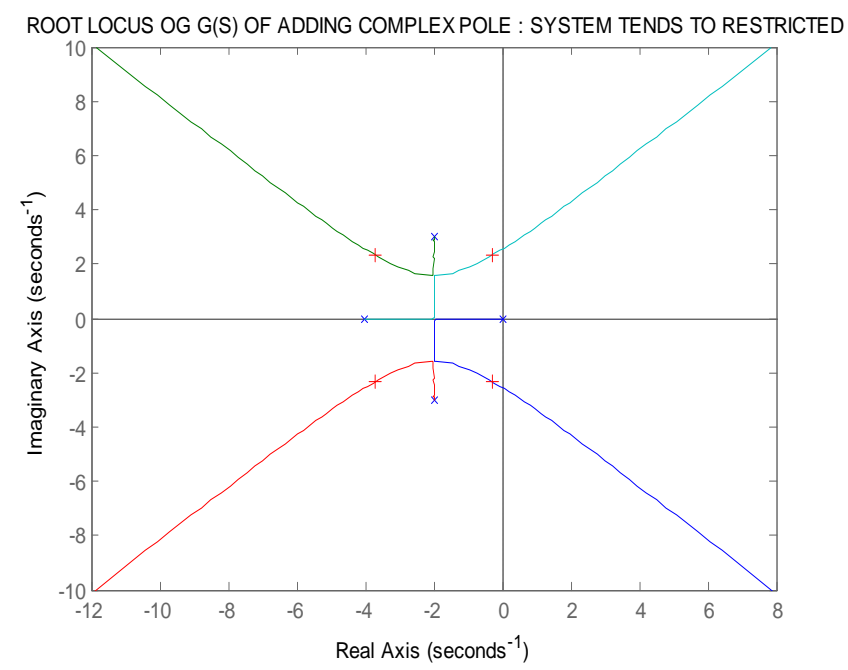

Select a point in the graphics window

selected_point $=0.6777+1.2112 \mathrm{i}: 107.7735$

For considering complex axis, for the constant gain parameter $0<\mathrm{k}_{3}<\mathrm{k}_{3}<\mathrm{k}_{2}<\mathrm{k}_{1}$ the root contour goes more beyond of origin towards right hand side which enhances instability but for addition the complex root in denominator system tends to 
became more restricted on stability and goes through L.H.S of s-plane.

Study Case2 : $\frac{k(s+a)}{(s+b)}=\frac{a d d}{\text { zero }}>\frac{k(s+a)(s+c)}{(s+b)}=$

\{Assuming, $|c|>|b|>|a|\} \frac{\text { add }}{\text { zero }}>\frac{k(s+a)(s+b)(s+c)}{(s+b)}$

Here the addition of zeros in the numerator indicates the left from origin that effects the improve of stability and for adding complex roots the stability is effected to reduce. Which is shown below :

\section{5 'Root Locus of $G(S)$ of Single Zero :In the Left}

Hand Side hence the System is Stable for all Values of $\mathbf{K}^{\prime}$

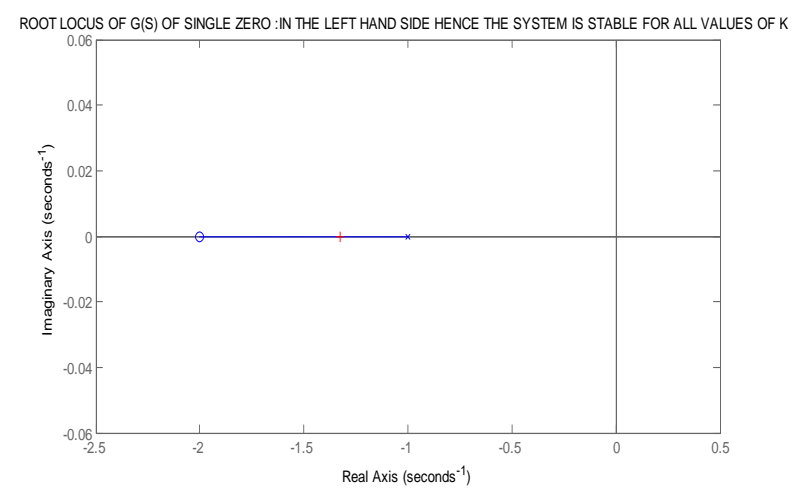

Select a point in the graphics window

selected_point $=-0.0665+0.0013 \mathrm{i}: 0.4828$

3.6 'Root Locus of G(S) with Two Zeros on the LHS of S Plane : In the Left Hand Side hence the System is More Stable for all Values of $K^{\prime}$

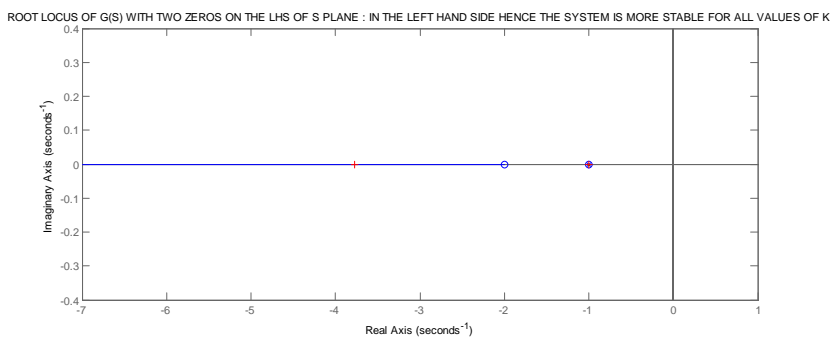

Select a point in the graphics window

selected_point $=-0.2227+0.0261 \mathrm{i}: \quad 0.5626$

\section{7 'Root Locus of G(S) with Increase of No of Zeors: System is Under Stable Totally'}

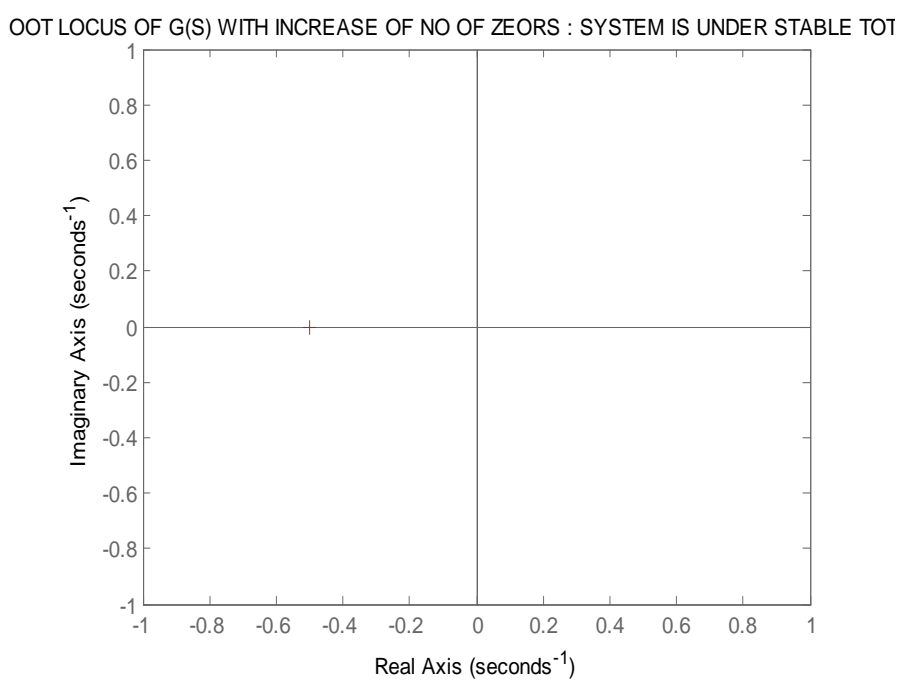

Select a point in the graphics window

selected_point $=0.3246+0.0466 \mathrm{i}: \quad 1$

\section{8 'Root Locus OG G(S) of Adding Complex Pole: System Stability Effected'}

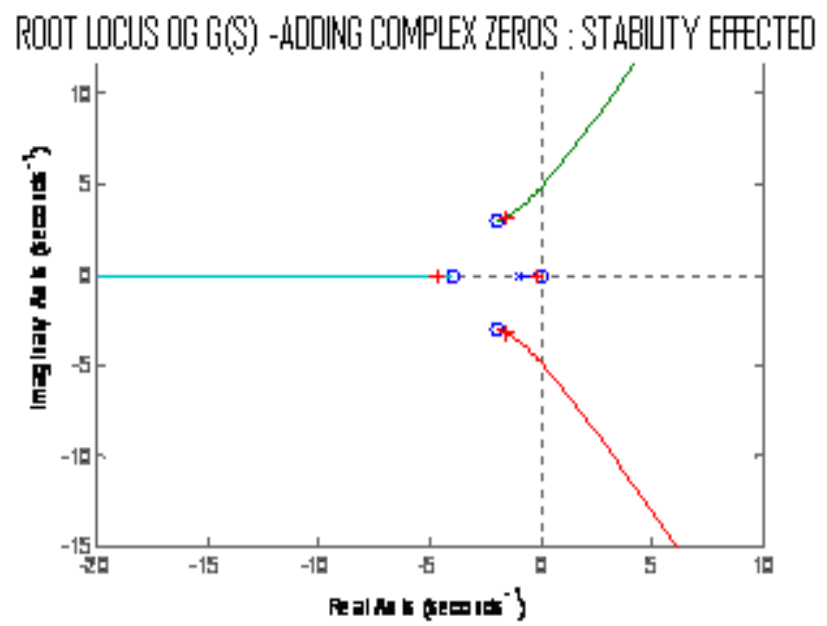

Select a point in the graphics window

selected_point $=-2.6185+2.9348 \mathrm{i}:$

0.0708

Now these all are bounding for linear system basically. For Non linear the system demands boundation for every harmonics. Practically this is very difficult. 


\section{NON LINEAR CONTROL WITH DESCRIBING FUNCTION IN MIMO NETWORK:}

Due to affects of harmonics present in this system, its better to represent the processing function by the complex ratio of the fundamentals harmonics present in this system to the input component.

\section{Processing Transfer Function :}

\section{$\frac{\mid \text { Amplitudes of the systems Harmonics } \mid}{\mid \text { Amplitudes of the inputs component } \mid}, \angle \theta$}

$\theta$ is the phase shift of harmonics w.r.t input components

Consider a system which is combined for a MIMO based system cascaded with dead zone saturation system with a negative unity feedback.

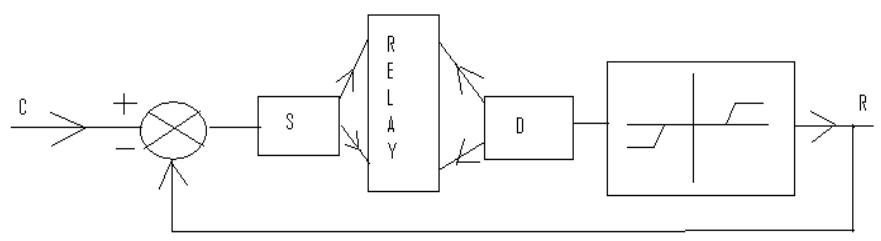

Fig 3: Model of MIMO based network with Non Linear Saturation System

Figure. 3 shows a wireless communication system where terminal $\mathrm{S}$ is communicating with terminal $\mathrm{D}$ through the relay terminal $R$. The relay $R$ is equipped with $(M=2)$ receiving antennas for reception of signal transmitted from terminal $\mathrm{S}$ and $(\mathrm{N}=2)$ transmitting antenna to convey the signal to terminal D after suitable amplification or decoding (as the case may be) at the Relay. The Relay is cascaded with Dead Zone Saturation Non Linear system. We assume that Maximum Ratio Combining (MRC) is used for receiving the signal at $\mathrm{R}$ and Maximum Ratio Transmission (MRT) is used for conveying it to the destination D. In MRC, [ 5 ] the branch with the instantaneous SNR is selected as the output of the combiner is given by :

$$
\gamma_{m r c}=\sum_{i=1}^{2} \gamma i
$$

Where the instantaneous signal-to-noise ratio (SNR) of the $\mathrm{i}^{\text {th }}$ branch is, : $\gamma_{i}=E_{s} / N_{0}\left[\alpha_{i}^{2}\right], \quad \mathrm{i}=1,2 \ldots$ With $\alpha_{i}$ being the Rayleigh fading amplitude of the channel

Between terminals $\mathrm{S}$ and the antennas $(\mathrm{i}=1,2)$ at relay $\mathrm{R}$, $E_{S}$ is the energy of the transmitted signals and $N_{0}$ is the onesided noise power spectral density per branch.
We assume the channel is frequency non-selective and slowly varying such that it is constant over the transmitted symbols interval. Noting that $\alpha_{i}$ is Rayleigh distributed, $\alpha_{i}^{2}$ is the exponentially distributed random variables. If we further assume that $(S N R)_{i}$ is the same for all diversity branches (i.e., $\overline{\gamma_{1}}=\overline{\gamma_{2}}$, the output SNR of the MRC combiner(or, the $\mathrm{SNR}$ between $\mathrm{S}$ and $\mathrm{R}, \gamma_{P, Q}$ ) has the probability density function (pdf) is given by

$$
\begin{aligned}
& \mathrm{p} \Gamma_{S, R}\left(\gamma_{S, R}\right)=\frac{\gamma_{S, R}^{2-1}}{\bar{\gamma}_{S}^{2} \Gamma(2)} e^{-\frac{\gamma_{S, R}}{\gamma_{S}}} \\
& \mathrm{p} \Gamma_{S, R}\left(\gamma_{S, R}\right)=\frac{\gamma_{S, R}^{1}}{\bar{\gamma}_{S}^{2} \Gamma(2)} e^{-\frac{\gamma_{S, R}}{\gamma_{S}}}
\end{aligned}
$$

Assuming $\alpha_{j}, \mathrm{j}=1$ and 2 the fading amplitude of channel between two antennas at $\mathrm{R}$ and $\mathrm{D}$ as Rayleigh type, the $(S N R)_{j}$ is the same for all diversity branches, namely $\overline{\gamma_{R}}$ similar way the pdf of $\Gamma_{R, D}$ can be written by :

$$
\begin{gathered}
\mathrm{p} \Gamma_{R, D}\left(\Gamma_{R, D}\right)=\frac{\gamma_{R, D}^{2-1}}{\bar{\gamma}_{D}^{2} \Gamma(2)} e^{-\frac{\gamma_{R, D}}{\bar{\gamma}_{R}}} \ldots \ldots(5) \\
\mathrm{p} \Gamma_{R, D}\left(\Gamma_{R, D}\right)=\frac{\gamma_{R, D}^{1}}{\bar{\gamma}_{D}^{2} \Gamma(2)} e^{-\frac{\gamma_{R, D}}{\bar{\gamma}_{R}}}
\end{gathered}
$$

Where $\overline{\gamma_{R}}$ is the average SNR of the $\mathrm{R}$ to $\mathrm{D}$ link.

Choosing the appropriate gain at the relay terminal before retransmission, the overall SNR at the receiving terminal R can be very closely upper bounded as,

$$
\gamma_{e q}=\frac{\Gamma_{S, R} \Gamma_{R, D}}{\Gamma_{S, R}+\Gamma_{R, D}}
$$

Where $\Gamma_{R, D}$ is the output SNR of the MRC, and $\Gamma_{R, D}$ is the instantaneous SNR between R and D with MRT

We also obtained [ 6 ],

$$
\int_{0}^{\infty} S^{k-1} 2 \sqrt{\beta} s k_{1}(\sqrt{\beta} s) d s=\frac{\Gamma(k+1) \Gamma(k)}{\beta^{k}}
$$

And hence reduce to: 


$$
M_{x}(\mathrm{~S})=\sum_{K}^{\propto}-0 \frac{(-S)^{K}}{\Gamma(K+1)}\left[\frac{\Gamma(K+1) \Gamma(K)}{\beta^{K} \Gamma(K)}=\frac{\beta}{S+\beta}\right.
$$

Using the above results for mgf of destination SNR one can derive the average bit error rate (ABER) of several digital modulation schemes over the proposed relayed link by adopting the mgf-based approach. For demonstration, we consider here the ABER of binary differential phase-shift keying (DPSK). For amplify-and - forward relaying the ABER is given by:

$$
P_{b}(e)=\frac{1}{2} M_{\Gamma}(1)
$$

For decode -and-forward relaying the ABER can be shown to be given by

$$
P_{b}(e)=P_{b}\left(e_{1}\right)+P_{b}\left(e_{2}\right)-2 P_{b}\left(e_{1}\right) P_{b}\left(e_{2}\right)
$$

Where $P_{b}\left(e_{1}\right)=$ the ABER for $\mathrm{S}$ to $\mathrm{R}$ link and is given by:

$$
\begin{aligned}
& P_{b}\left(e_{1}\right)=\int_{0}^{\infty} \frac{1}{2} e^{-\gamma / s, r} p_{T_{s, r}}\left(\gamma_{s,}, d \gamma_{S, r}\right. \\
& P_{b}\left(e_{1}\right)=\frac{1}{2}\left(\frac{\beta_{1}}{1+\beta_{1}}\right)^{2} .
\end{aligned}
$$

Similarly the ABER for R to D link and is obtained as,

$$
P_{b}\left(e_{2}\right)=\frac{1}{2}\left(\frac{\beta_{2}}{1+\beta_{2}}\right)^{2}
$$

The ABER for decode - and forward relaying can be written as:

$P_{b}(e)=\frac{1}{2}\left(\frac{\beta_{1}}{1+\beta_{1}}\right)^{2}+\frac{1}{2}\left(\frac{\beta_{2}}{1+\beta_{2}}\right)^{2}-$

$2 \times \frac{1}{2}\left(\frac{\beta_{1}}{1+\beta_{1}}\right)^{2} \times \frac{1}{2}\left(\frac{\beta_{2}}{1+\beta_{2}}\right)^{2}$

For symmetric case, with $\beta_{1}=\beta_{2}=\beta=\frac{1}{\bar{\gamma}}$ may be reduced to

$$
P_{b}(e)=\frac{1}{2}\left[2\left\{\frac{1}{(1+\bar{\gamma})}\right\}^{2}-\left\{\frac{1}{(1+\bar{\gamma})}\right\}^{4}\right]
$$

The ABER curves for decode-and-forward (DF) relaying over the proposed relay over Rayleigh fading channel with respect to the normalized SNR. The horizontal axis represents the instantaneous SNR in $\mathrm{dB}$ and the vertical axis represents the average bit error rate (ABER). Observations are noted for the cases of decode-and-forward relaying systems from the plots of Fig.4, where improvements are even less. This nature of the graph is highly expected because, with diversity, merely $\mathrm{S}$ $\rightarrow \square \mathrm{R}$ link SNR is improved whereas overall performance is determined by both $\mathrm{S} \rightarrow \square \mathrm{R}$ and $\mathrm{R} \rightarrow \mathrm{D}$ links The $(S / N)$ ratio is improved for $\mathrm{M}=2$ and $\mathrm{N}=2$.

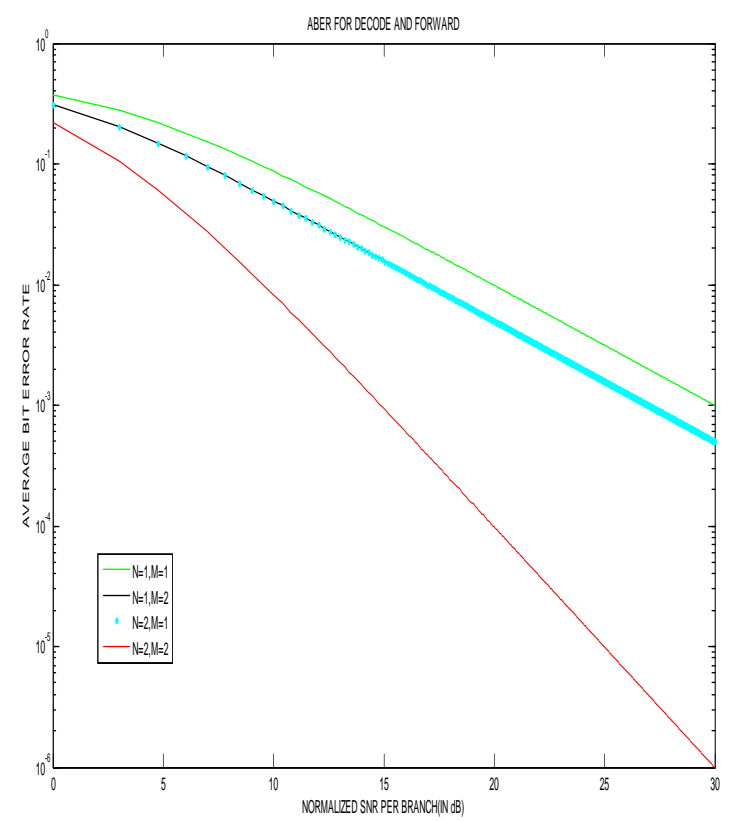

Fig 4 : Plot of ABER verses SNR (dB) for decode and forward relaying with transmitting antenna $\mathrm{M}=1, \mathrm{~N}=1 ; \mathrm{M}=1, \mathrm{~N}=2 ; \mathrm{M}=2, \mathrm{~N}=1 ; \mathrm{M}=2, \mathrm{~N}=2$.

Now the output of this MIMO network is passing through the dead zone saturation system and the corresponding diagram is shown below : 


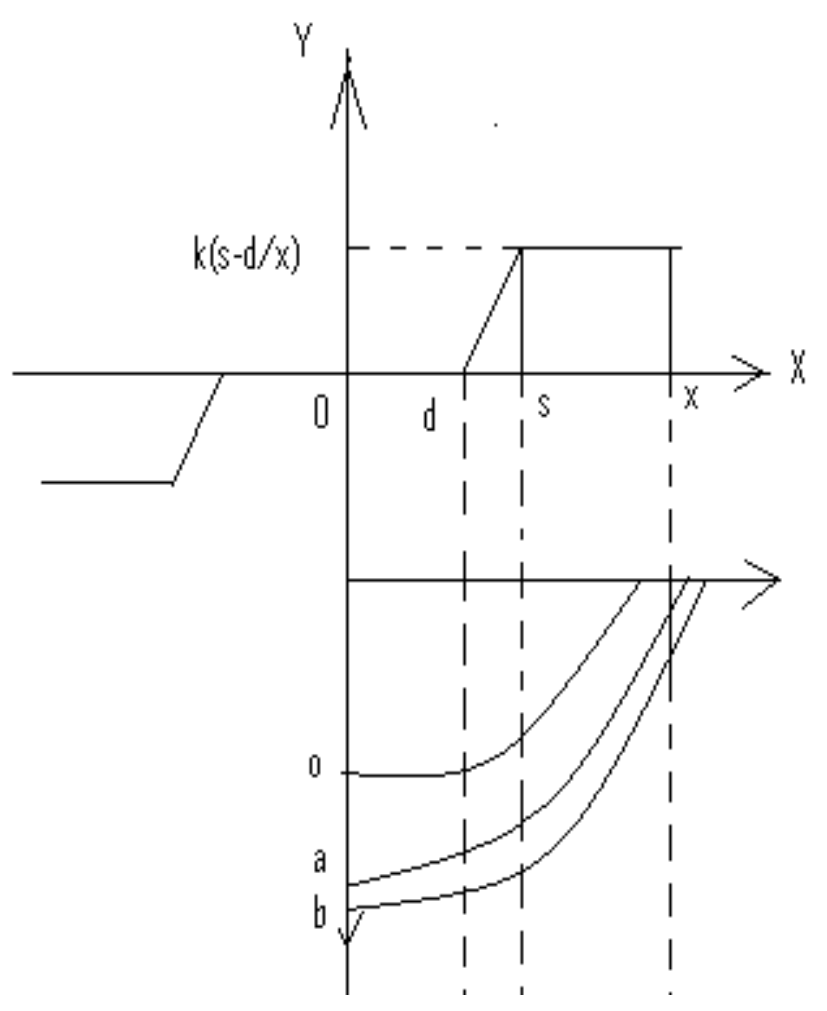

Here in between the region ' $d$ ' -> ' $x$ ' , the slope is $k$, so the portion of the output of MIMO network over ABER vs $S / N$ ratio shows linear characteristics, before the ' $d$ ' i.e $0<x<d$. the output is dead zone and after $\mathrm{s}<\mathrm{x}<\infty$ the output $=k(s-d / x)$,

So the improvement of Signal to Noise Ratio is somehow hampering for some time period span, that's affects the system is unstable condition. Now if we connect a Zero Order Hold circuit the stability is improvement that we see via simulink design.

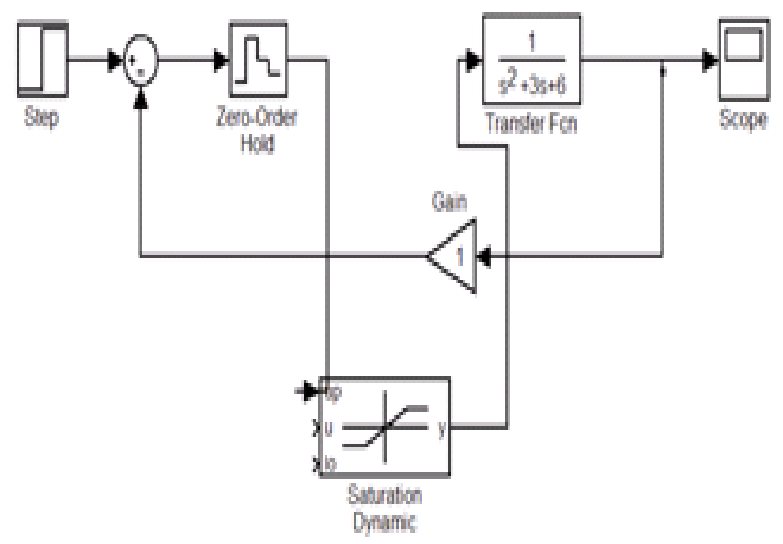

Fig 5(a): $\mathrm{ZOH}$ connected in saturation system

\section{Output:}
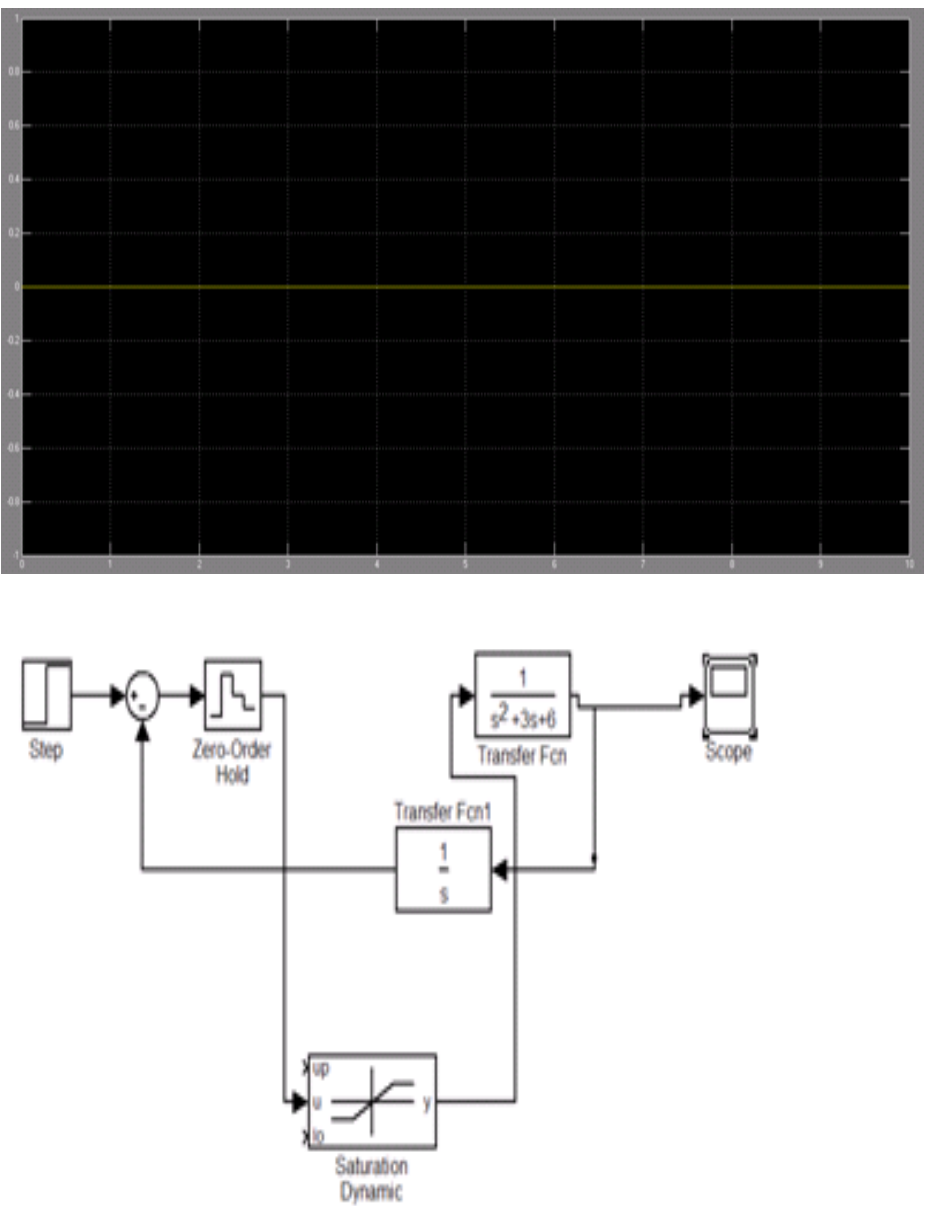

Fig 5(b): ZOH connected in saturation system with step input and Step feedback processing function

\section{Output:}

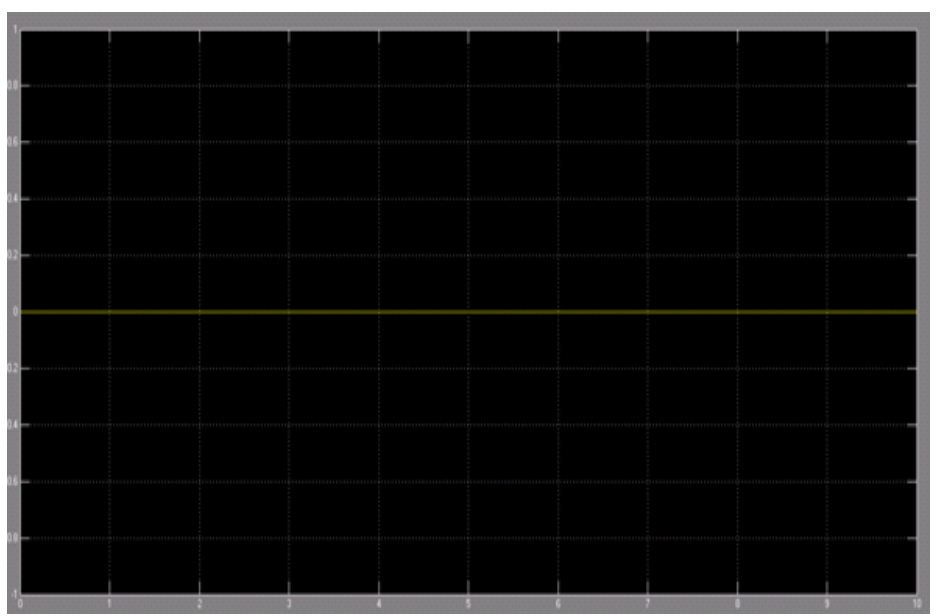




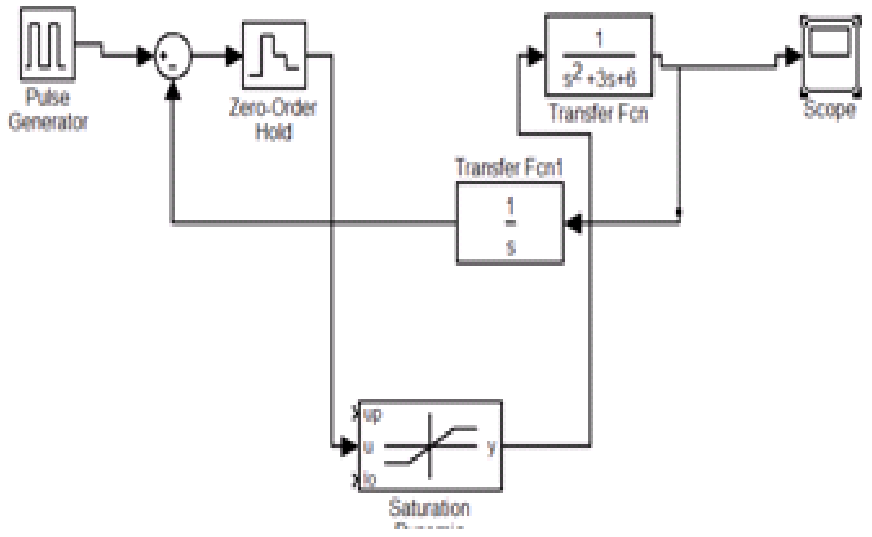

Fig 5(c): ZOH connected in saturation system with pulse input and Step feedback processing function

\section{Output:}

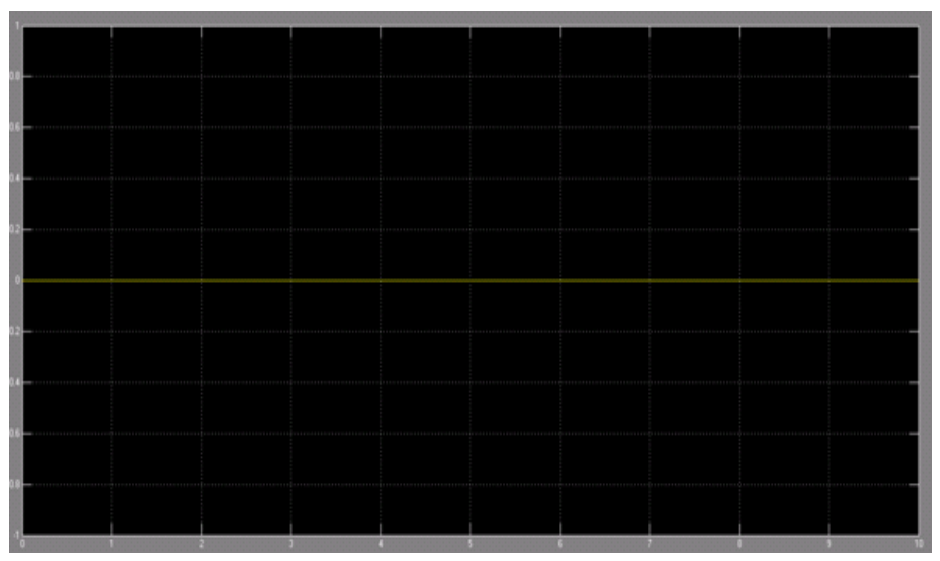

Now if the system is disturbed by some external trigger the system's stability is hampering. For example consider the following diagram for a sinusoidal input with an external trigger input. Here the summer output connect below portion of saturation level

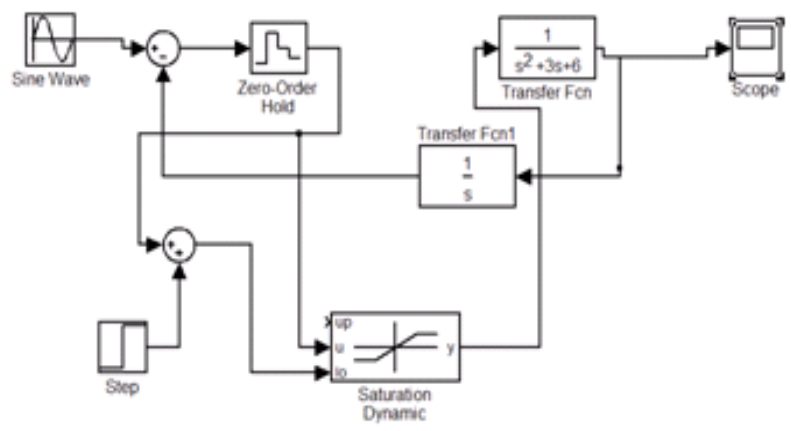

Fig 5(d): $\mathrm{ZOH}$ connected in saturation system with sinusoidal input with external trigger input and Step feedback processing function

\section{Output:}

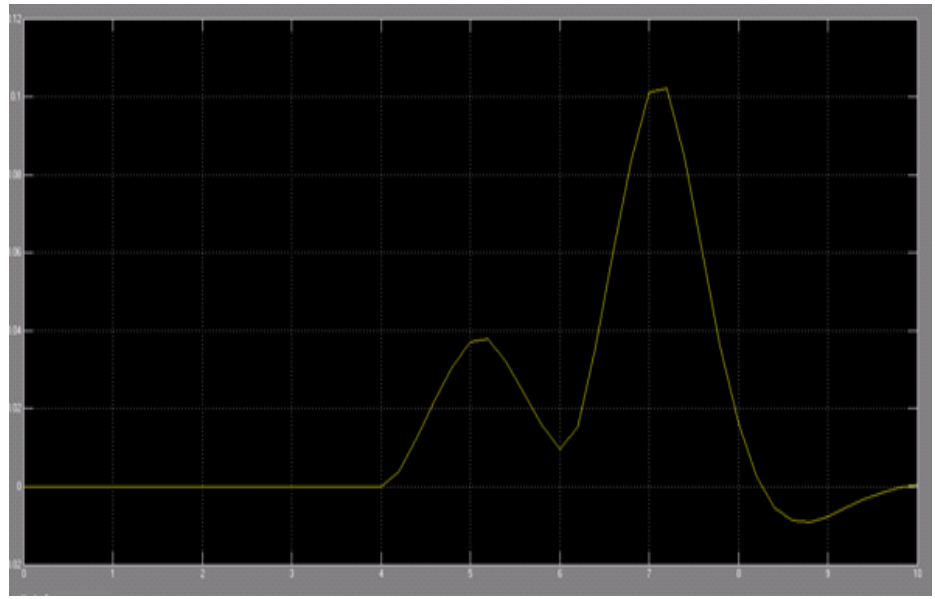

Now if the summer output connect up portion of saturation level then the stability is unchanged though the external trigger is connected.

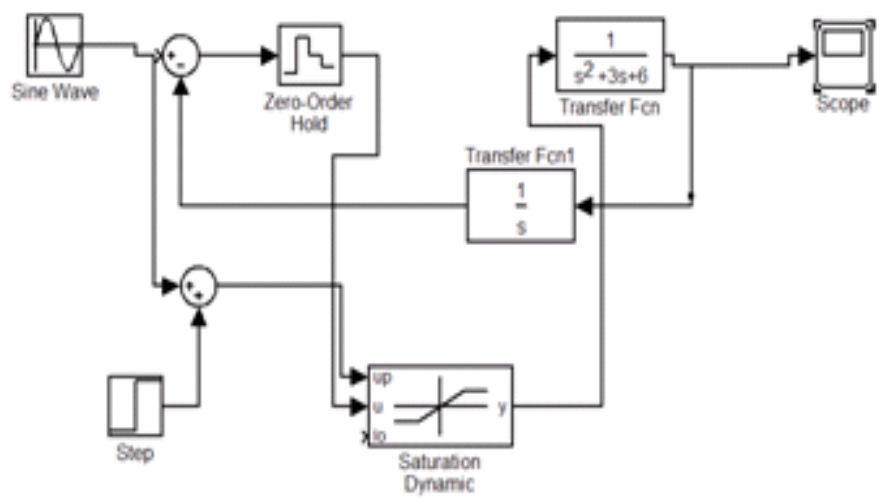

Figure 5(e): $\mathrm{ZOH}$ connected in saturation system with sinusoidal input with external trigger input and Step feedback processing function : Summer output in up portion of saturation

\section{Output:}

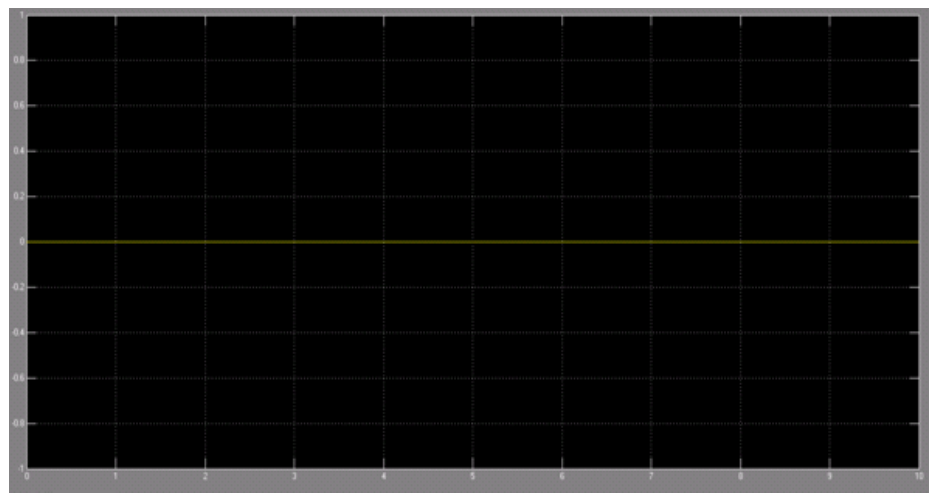




\section{CONCLUSIONS}

From the above diagrams we see the effects of Zero Order Hold technique for smoothly running a system. Basically Nonlinearities affects a system beyond of boundary. For that the Region of Convergence of that system shows uncertainty condition. This paper has given a brief overview of some of the simpler strategies for nonlinear control. Some of these methods, e.g., feedback linearization can be seen to be very close to linear methods and, as such, are easy to use in practice.

\section{ACKNOWLEDGMENTS}

The authors would like to thank the authorities of Birbhum Institute of Engineering and Technology for providing every kind of supports and encouragement during the working process. The authors thanks to reviewers for giving us such attention and time. The authors also acknowledge the unknown referees for their valuable comments and suggestions for improvement. Last but not the least the authors are giving a vote of thanks to our nearest and dearest parents and our be loving family members for providing mentally support to us.

\section{REFERENCES}

[1]. Non Linear Control Theory Introduction: Harry G.Kwatny , Department of Mechanical Engineering \& Mechanics , Drexel University

[2]. Control of Nonlinear Dynamic Systems: Theory and Applications: J. K. Hedrick and A. Girard () 2010

[3]. A Brief Overview of Nonlinear Control : Graham C. Goodwin (With Jose De Dona, Osvaldo J. Rojas and Michel Perrier) Centre for Integrated Dynamics and Control Department of Electrical and Computer Engineering The University of Newcastle NSW 2308 Australia1

[4]. Modern Control Systems, Dorf and Bishop, $7^{\text {th }}$ Edition, Addison-Wesley, 1995.

[5]. M.O. Hasna and M.-S.Alouini, "End-to-end performance of transmission systems with relays over Rayleigh-fading channels," IEEE Trans .Wireless Communication, vol. 2, no. 6, pp. 1126-1131, Nov. 2003

[6]. I. S. Gradshteyn and I. M. Rzyhik, Table of Integrals, Series and Products, $5^{\text {th }}$ ed. Academic Press, 1994

[7]. MATLAB and SIMULINK, The Math Works, Inc., 24 Prime Park Way, Natick, MA 01760.

[8]. O.J. Rojas and G.C. Goodwin, "A nonlinear control strategy related to feedback linearization capable of dealing with non-stably invertible nonlinear systems,"Technical Note, Univ. of Newcastle, 2001.

\section{BIOGRAPHIES}

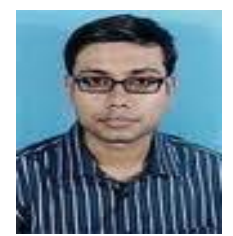

Nirmalya Chandra was born in India. $\mathrm{He}$ received the B.Tech and $\mathrm{M}$.Tech degree in Electronics and Communication from the West Bengal University of Technology, Kolkata in 2007 and 2010 respectively.He has been an Assistant Professor in the Department of Electronics and Communication Engineering, Birbhum Institute of Engineering and Technology ( Govt. Aided Institution ), Suri, Birbhum-731101. His currently research interest in Control System, Adhoc Networks, Digital Modulation, Digital Signal Processing etc. Email : chandra.nirmalya@gmail.com

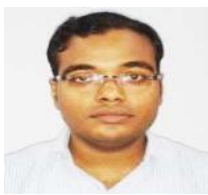

Samiran Maiti was born in India and obtained his M.Tech degree from University college of Science\& Technology, Calcutta Univesity, Calcutta, West Bengal. Now he is Asst. Professor of E.C.E. Department in Birbhum Institute of Engineering \& Technology,P.O.: Suri, Dist: Birbhum, Pin: 731101, West Bengal, India. His research interests include control engineering, Microcontroller based system design, instrumentation, Biomedical Engineering and Digital signal processing. E-mail: samiran.cemk@gmail.com

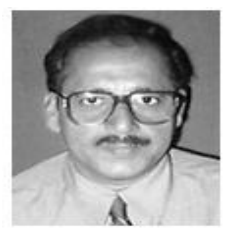

Dr. Achintya Das was born on February 8, 1957. He received the $M$. Tech. and Ph.D. (Tech.) degrees in Radio Physics and Electronics from the University of Calcutta, Calcutta, India, in 1982 and 1996, respectively. He was an Executive of Quality Assurance with Philips India from 1982 to 1996 . He is currently a Professor and Head of Electronics and Communication Engineering Dept. at Kalyani Government Engineering College, Kalyani, Nadia, West Bengal, India. His research interests include control engineering, instrumentation, Biomedical Engineering and signal processing. He is reviewer of International Journal of Control, England. He has sixty five publications in national Journals and national / international / world conferences. Special awards he received are i). Gold Medal for securing highest position with 1st class in M.Tech. (Calcutta University) and Mohallanobis Medal Award for securing highest position with $1^{\text {st }}$. class in P.G.Dip in SQC (IAPQR, Delhi). He has more than 80 research publications published in National/ International journal, National/ International world conference 